\title{
A New Theorem on Bargaining Sets in TU Games
}

\author{
Wenbo Yang ${ }^{1, a}$, Jiuqiang Liu $^{2, b}$ \\ ${ }^{1}$ Department of Electrical Engineering and Automation, Luoyang Institute of Science and \\ Technology, Luoyang, 471023, China \\ ${ }^{2}$ Department of Mathematics, Eastern Michigan University, Ypsilanti, MI 48197, USA \\ aemail: ywb1029@163.com, bemail: jliu@163.com
}

Keywords: Soccer Robot; Mechanical Analysis; Optimal Design

Abstract. In this paper, we provide a new existence theorem by proving that Mas-Colell bargaining sets exist for all TU games.

\section{Introduction}

Let $N=\{1,2, \ldots, n\}$ be the set of $n$ players. Any subset of $\mathrm{N}$ is called a coalition.

Definition 1.1. A cooperative game (or a TU game) in characteristic function form with player set $N$ is a map $v: 2^{\mathrm{N}} \rightarrow \square \quad$ with the property $v(\phi)=0$.

A payoff vector $x \in \square^{n}$ is said to be individual rational if $x_{i} \geq v(\{i\})$ for each $i \in N$.

Definition 1.2. The imputation set $I(v)$ of a cooperative game $v$ is the set

$$
I(v)=\left\{x \in \square^{n} \mid \sum_{i \in N} x_{i}=v(N), x_{i} \geq v(\{i\}) \text { for each } i \in N\right\}
$$

Cooperative games have been studied extensively in the literature. A central question in cooperative games is to study solution concepts and their relationships, those well-known solution concepts include cores, stable sets, Shapley values, bargaining sets, and so on.

To state Vohra's result formally, let us recall some necessary concepts from [4].

A non-transferable utility game (NTU game) in characteristic function form is defined as a pair $(N, V)$, where $V: 2^{N} \rightarrow \square^{N}$ is a correspondence satisfying

(i) for all non-empty $S \in 2^{N}, V(S)$ is non-empty, closed, and comprehensive,

(ii) for all $i \in N, V(\{i\})=\left\{x \in \square^{N} \mid x_{i} \leq 0\right\}$,

(iii) for all $S \in 2^{N}, V(S)_{S} \cap \square_{+}^{s}$ is bounded.

A TU game $v$ in characteristic function form is equivalent to an NTU game $(N, V)$ such that for every non-empty $S \in 2^{N}$,

$$
V(S)=\left\{x \in \square^{N} \mid \sum_{i \in S} x_{i} \leq v(S)\right\} .
$$

In fact, Condition (ii) in the definition above by Vohra also requires $v(\{i\})=0$ for all $i \in N$, which can be achieved by zero normalization.

Weak Superadditivity (version 1): For any $S \in 2^{N}$ and $i \notin S$, if $x \in V(S)$, then $y \in V(S \bigcup\{i\})$, where $y_{i}=0$ and $y_{j}=x_{j}$ for $j \neq i$.

This has the following equivalent form given in [2].

Weak Superadditivity (version 2): An NTU game $(N, V)$ is weakly superadditive if for every $i \in N$ and every $S \subseteq N \backslash\{i\}$ satisfying $S \neq \phi, V(S) \times V(\{i\}) \subseteq V(S \cup\{i\})$.

Clearly, for TU games, the weak superadditivity is equivalent to the following according to version 2 and (1.1). 
Weak Superadditivity for TU games: $v(S)+v(\{i\}) \leq v(S \cup\{i\})$ for each $S \subseteq N$ and each $i \in N \backslash S$.

In 1991, Vohra [4] proved the following existence theorem for Mas-Colell bargaining sets.

Theorem 1.3 (Vohra, 1991). If $v$ is a weakly superadditive TU game, then the Mas-Colell bargaining set $M B(v)$ of $v$ is non-empty.

In this paper, we prove the following stronger existence theorem for Mas-Colell bargaining sets in TU games.

Theorem 1.4. If $v$ is a TU game such that $v(S) \leq v(N)$ for each $S \subseteq N$, then the Mas-Colell bargaining set $M B(v)$ of $v$ is non-empty.

Lemma 1.7. Let $v$ be a TU game and let $v_{0}$ be the zero-normalized game of $v$. Then $x \in M B(v)$ if and only if $x^{\prime} \in M B\left(v_{0}\right)$, where $x_{i}^{\prime}=x_{i}-v(i)$ for each $i \in N$.

\section{Proof of Theorem 1.4}

In this section, we will give a proof for Theorem 1.4 by proving the following Theorem 2.2 which implies Theorem 1.4. Our proof is motivated in part by the ideas from [4] and [5]. Let $v$ be a TU game. For an imputation $x \in I(v)$ and a coalition $S \subseteq N$, the excess of $\mathrm{S}$ at $x$ is

$$
e(S, x)=v(S)-\sum_{k \in S} x_{k}
$$

Clearly, we have following remark from the definitions.

Remark 2.1. An objection $(S, y)$ at $x$ exists if and only if $e(S, x)>0$.

Next, for the purpose of overcoming difficulties in our proof for Theorem 1.4, we in- troduce strong counterobjection as follows, where the special conditions imposed on strong counterobjection is just a technical device.

Strong Counterobjection: Given an objection $(S, y)$ at $x \in I(v)$, a strong counterobjection to $(S, y)$ at $\mathrm{x}$ is a pair $(T, \mathrm{z})$, where $T$ is a coalition such that $T \backslash S \neq \phi$ and there exists $h \in S \backslash T$ satisfying $y_{h}-x_{h}=\max \left\{y_{i}-x_{i} \mid i \in S\right\}>0$, and $z$ is a vector in $R^{T}$ satisfying that $z(T)=\sum_{i \in T} z_{i}=v(T), z_{i} \geq y_{i}$ for each $i \in S \cap T$, and $z_{i} \geq x_{i}+\frac{\sum_{j \in S \backslash T}\left(y_{j}-x_{j}\right)}{|T \backslash S|}$ for each $i \in T \backslash S$.

An imputation $x \in I(v)$ is said to belong to strong Mas-Colell bargaining set $M B_{S}(v)$ if for any objection $(S, y)$ at $x$, there exists a strong counterobjection to it at $x$.

Theorem 2.2. If $v$ is a TU game such that $v(S) \leq v(N)$ for each $S \subseteq N$, then the strong Mas-Colell bargaining set $M B_{S}(v)$ of $v$ is non-empty.

Lemma 2.3. Given an objection $(S, y)$ at $x$ and a non-empty coalition $T$ such that $T \backslash S \neq \phi$ and there exists $h \in S \backslash \mathrm{T}$ satisfying $y_{h}-y_{h}=\max \left\{y_{i}-x_{i} \mid i \in S\right\}>0$, then a strong counterobjection $(T, z)$ to $(S, y)$ at $x$ exists if and only if $e(T, x) \geq e(S, x)$.

Next we introduce the concept of balanced collection and a result from [11] which is needed in our proof.

Let $\Delta^{N}$ be the standard simplex:

$$
\Delta^{N}=\left\{x \in R^{N} \mid x_{i} \geq 0 \text { for each } i \in N \text { and } \sum_{i=1}^{n} x_{i}=1\right\} .
$$

Its $i$-th face is $\Delta^{N \backslash\{i\}}=\left\{x \in \Delta^{N} \mid x_{i}=0\right\}$. For each $S \subseteq N$, denote $e^{S}$ the n-dimensional vector 
with $e_{i}^{S}=1$ if $i \in S$ and $e_{i}^{S}=0$ if $i \notin S$.

Definition 2.4. A collection $B$ of non-empty subsets (coalitions) of $N$ is balanced if there exist positive numbers $\lambda_{\mathrm{S}}$ for $S \in B$ such that

$$
\sum_{S \in B} \lambda_{s} e^{S}=e^{N}
$$

The numbers $\lambda_{\mathrm{s}}$ are called balancing coef $\mathrm{f}$ icients.

Clearly, the condition in (2.1) for a balanced collection $B$ is equivalent to the following.

$$
\sum_{S \in B: i \in S} \lambda s=1 \text { for each } i \in N \text {. }
$$

The next theorem is proved by Zhou.

Theorem 2.5 (Zhou, 1994). If $\left\{O_{S}\right\}_{S \in N}$ is a family of open sets of $\Delta^{N}$ that satisfy

(1) $\Delta^{N \backslash\{i\}} \subseteq O_{\{i\}}$ for each $i \in N$ and

(2) $\bigcup_{S \in N} O_{S}=\Delta^{N}$,

then there is a balanced collection $B$ of non-empty subsets ( coalitions ) of $N$ such that $\bigcap_{S \in B} O_{S} \neq \phi$.

Let $v$ be a TU game. Note that the core $C(v)$ of $v$ consists of all $x \in I(v)$ such that $e(S, x) \leq 0$ for all $S \subseteq N$. It follows from Remark 2.1 that the core $C(v)$ is a subset of Mas-Colell bargaining set $M B(v)$. Thus, whenever $v$ has a non-empty core, $M B(v)$ is nonempty. This means that, when we deal with the existence of $M B(v)$, we may assume that $C(v)=\phi$, that is, for any $x \in I(v)$, there exists $S \subseteq N$ such that $e(S, x)>0$. For each $x \in I(v)$, let $e_{x}=\min \{e(S, x) \mid S \subseteq N$ with $e(S, x)>0\}$ and set

$$
\varepsilon_{x}=\min \left\{\frac{1}{n} e_{x}, \frac{1}{n} v(N)\right\}
$$

Then, under the assumption that $v(N)>0$ and $C(v)=\phi, \varepsilon_{x}>0$ for each $x \in I(v)$.

Let $v$ be a TU game and $x \in I(v)$. We say an objection $(S, y)$ at $x$ is strongly justif-ied if there is no strong counterobjection to $(S, y)$ at $x$. For each non-empty $S \subseteq N$, define $O_{S}$

as follows:

$$
\begin{aligned}
& O_{\{i\}}=\left\{x \in I(v) \mid x_{i}<\varepsilon_{x}\right\} \text { for each } i \in N, \\
& O_{S}=\{x \in I(v) \mid \text { there exists a strongly justified objection }(S, y) \text { at } x\} \text { if }|S| \geq 2 .
\end{aligned}
$$

The following fact follows from the definition immediately.

Fact 2.6. Let $v$ be a TU game with empty core and $v(N)>0$. For each $i \in N, \Delta^{N \backslash\{i\}} \subseteq O_{\{i\}}$.

Lemma 2.7. Let $v$ be a TU game with $v(N)>0$. Then, for each non-empty $S \subseteq N, O_{S}$ is open.

Lemma 2.8. Let $v$ be a TU game such that $v(N)>0$ and $v(\mathrm{~S}) \leq \mathrm{v}(\mathrm{N})$ for each $\mathrm{S} \subseteq N$. Then for any balanced collection $B$ of coalitions, $\bigcap_{S \in B} O_{s}=0$.

The next lemma allows us to assume $\mathrm{v}(\mathrm{N})>0$ when dealing with the non-emptiness of strong Mas-Colell bargaining sets.

Lemma 2.9. Let $v$ be a TU game and let $b>0$ be such that $\mathrm{v}(\mathrm{N})+b>0$. Define $v$ ' to be the game such that $v^{\prime}(S)=v(S)+\frac{|s|}{n} b$ for each $\mathrm{S} \subseteq \mathrm{N}$. Then $\mathrm{x} \in M B_{S}(\mathrm{v})$ if and only if 
$x^{\prime} \in M B_{s}\left(v^{\prime}\right)$ where $x_{i}^{\prime}=x_{i}+\frac{b}{n}$ for each $i \in N$.

We now prove Theorem 1.4 by proving Theorem 2.2.

Proof of Theorem 2.2. Let $v$ be a TU game such that $\mathrm{v}(\mathrm{S}) \leq \mathrm{v}(\mathrm{N})$ for all $\mathrm{S} \subseteq N$. In view of Lemma 2.9, we may assume $v(N)>0$. In fact, if $\mathrm{v}(\mathrm{N}) \leq 0$, then let $\mathrm{b}>0$ be such that $\mathrm{v}(\mathrm{N})+\mathrm{b}>$ 0 and define $v^{\prime}$ to be the game such that $v^{\prime}(S)=v(S)+\frac{|s|}{n} b$ for each $S \subseteq \mathrm{N}$. Then $v^{\prime}(\mathrm{N})=\mathrm{v}(\mathrm{N})+$ $\mathrm{b}>0$ and $\mathrm{v}^{\prime}(\mathrm{S}) \leq \mathrm{v}^{\prime}(\mathrm{N})$ for each $\mathrm{S} \subseteq \mathrm{N}$. By Lemma 2.9, $M B_{s}(v)$ is non-empty if and only if $M B_{s}\left(v^{\prime}\right)$ is non-empty. Thus, we may assume $\mathrm{v}(\mathrm{N})>0$. If the core $\mathrm{C}(\mathrm{v})$ of $\mathrm{v}$ is non-empty, then we have the strong Mas-Colell bargaining set $M B_{s}(v)$ is non-empty. Thus, we may assume that the core $C(\mathrm{v})$ is empty.

Recall that for each $\mathrm{x} \in \mathrm{I}(\mathrm{v}), \sum_{i=1}^{n} x_{i}=v(N)>0$. We map $\mathrm{Q}=\mathrm{I}(\mathrm{v})$ onto the standard simplex $\Delta^{N}$ by $f$ :

$$
f: x \rightarrow \frac{x}{\sum_{i=1}^{n} x_{i}}
$$

Suppose, to the contrary, that the strong Mas-Colell bargaining set $M B_{s}(v)$ is empty. Then we have $Q \backslash \mathrm{U}_{0} \neq{ }_{s \subseteq N} O{ }_{s}=0$. This means that $\Delta^{N}=\mathrm{f}(\mathrm{Q})=\mathrm{U}_{0} \neq{ }_{s \subseteq N} f\left(O_{s}\right)$. By Fact 2.6, $\square^{N \backslash\{i\}} \subseteq f\left(O_{\{i\}}\right)$ for each i $\in$ N. It follows from Theorem 2.5 that there is a balanced collection B of coalitions such that $\bigcap_{s \in B} f\left(\mathrm{O}_{s}\right) \neq 0$. But, by Lemma 2.8, we have

$\bigcap_{S \in B} O_{s}=\phi$.It follows that $\bigcap_{S \in B} f\left(O_{s}\right)=\phi$, a contradiction.thus, the theorem holds.

\section{Conclusion}

In this paper, we proofed a stronger existence theorem by proving that Mas-Colell bargaining sets exist for all TU games.

\section{References}

[1] R. J. Auman and M. Maschler, “The bargaining sets for cooperative games” Advances in Game Theory, and AW Tucker, Princeton University Press, Princeton, N.J., (1964), 443-476.

[2] B. Peleg and P. Sudholter, “On the non-emptyness of the Mas-Colell bargaining set”, Journal of Math Econ (2005) Vol. 41, 1060-1068.

[3] Ken-Ichi Shimomura, “Quasi-Cores in Bargaining Sets”, International Journal of Game Theory (1997) Vol. 26, 283-302.

[4] R. Vohra, “An existence theorem for a bargaining set”, J of Math Econ (1991) Vol. 20, 19-34.

[5] L. Zhou, “A New Bargaining Set of an N-Person Game and Endogenous Coalition”,Games and Economic Behavior (1994) Vol. 6, 512-526. 